\title{
Virtual reality improves the accuracy of simulated preoperative planning in temporal bones: a feasibility and validation study
}

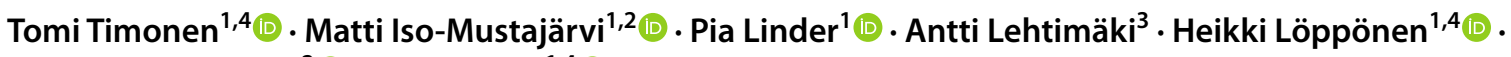 \\ Antti-Pekka Elomaa ${ }^{2} \cdot$ Aarno Dietz $^{1,4} \mathbb{1}$
}

Received: 24 June 2020 / Accepted: 8 September 2020 / Published online: 22 September 2020

(c) The Author(s) 2020

\begin{abstract}
Purpose Consumer-grade virtual reality (VR) has recently enabled various medical applications, but more evidence supporting their validity is needed. We investigated the accuracy of simulated surgical planning in a VR environment (VR) with temporal bones and compared it to conventional cross-sectional image viewing in picture archiving and communication system (PACS) interface.

Methods Five experienced otologic surgeons measured significant anatomic structures and fiducials on five fresh-frozen cadaveric temporal bones in VR and cross-sectional viewing. Primary image data were acquired by computed tomography. In total, 275 anatomical landmark measurements and 250 measurements of the distance between fiducials were obtained with both methods. Distance measurements between the fiducials were confirmed by physical measurement obtained by Vernier caliper. The experts evaluated the subjective validity of both methods on a 5-point Likert scale qualitative survey. Results A strong correlation based on intraclass coefficient was found between the methods on both the anatomical $(r>0.900)$ and fiducial measurements $(r>0.916)$. Two-tailed paired t-test and Bland-Altman plots demonstrated high equivalences between the VR and cross-sectional viewing with mean differences of $1.9 \%(p=0.396)$ and $0.472 \mathrm{~mm}(p=0.065)$ for anatomical and fiducial measurements, respectively. Gross measurement errors due to the misidentification of fiducials occurred more frequently in the cross-sectional viewing. The mean face and content validity rating for VR were significantly better compared to cross-sectional viewing (total mean score 4.11 vs $3.39, p<0.001$ ).

Conclusion Our study supports good accuracy and reliability of VR environment for simulated surgical planning in temporal bones compared to conventional cross-sectional visualization.
\end{abstract}

Keywords Virtual reality $\cdot$ Two-dimensional $\cdot$ Three-dimensional $\cdot$ Surgical planning $\cdot$ Temporal bone

\section{Introduction}

Tomi Timonen and Matti Iso-Mustajärvi equal contribution.

Electronic supplementary material The online version of this article (https://doi.org/10.1007/s00405-020-06360-6) contains supplementary material, which is available to authorized users.

Tomi Timonen

tomi.timonen@kuh.fi

1 Department of Otorhinolaryngology, Kuopio University Hospital, Puijonlaaksontie 2, PL 100, 70210 Kuopio, Finland

2 Microsurgery Centre of Eastern Finland, Kuopio, Finland

3 Department of Radiology, Kuopio University Hospital, Kuopio, Finland

4 School of Medicine, Institute of Clinical Medicine, University of Eastern Finland, Kuopio, Finland
One key issue for successful and safe surgery is thorough preoperative planning. The foundation for detailed preoperative planning of surgical procedures is based on individual image data and should be considered mandatory for modern operative care. In otolaryngology, preoperative imaging is mostly performed with computed tomography (CT) and magnetic resonance imaging (MRI). The image-stack manipulation and measurements are usually based on picture archiving and communication system (PACS) tools and volume renderings. These limit the image view to two-dimensional (2D) cross-sections and screens, missing stereoscopy and freedom of dimensional control. Despite the advances in preoperative imaging, images are still examined in $2 \mathrm{D}$ cross sections thus requiring the surgeon to construct and 
formulate an understanding of complex three-dimensional relationships. This is challenging not only for novice surgeons but also for experienced surgeons in complex cases. Recent advances in virtual reality (VR) technologies have yielded numerous VR applications for surgical planning, which may overcome these restrictions [1]. These new VR applications offer promising tools for surgical training and preoperative planning [2-4].

The first medical VR applications were introduced in the 1990s. They mainly focused on visualization of complex anatomy, preoperative planning, surgery training and telemedicine [5]. In recent years, the availability of consumergrade VR technology has re-emerged the interest for medical use. The VR is a stereoscopic three-dimensional (3D) computer-generated environment, which provides an interactive stereoscopic 3D view of objects. The development of head-mounted displays and hand-held controllers with motion tracking sensors provide users with a versatility and a possibility to approach the multidimensional anatomy of the patient at any possible angle. Users can freely control magnification, windowing of image parameters and mark or paint objects in the image view. State-of-the-art VR systems already reproduce 3D anatomy to a high level of immersion and authenticity not achievable with conventional cross-sectional 2D images and thus may contribute to a better understanding of the anatomy in question [6]. The VR environment allows users to perceive critical anatomical landmarks and their relationship in the same virtual space, which adds to better memory recall compared to traditional 2D screen interface [7]. However, nausea, vertigo and headache have been reported with VR in $30-80 \%$ of the users, depending on the software $[8,9]$.

VR surgical planning is gaining increasing attention since it has been shown to augment operative accuracy, efficiency and outcomes [10-12]. However, there are only very few independent studies, which investigate the validity and accuracy of VR in authentic settings [13]. Best practice requires that new medical applications such as the VR surgical planning software are tested for subjective and objective validity [14]. Subjective validity is commonly evaluated with the face and content validity via different acceptance surveys by experts in the field. The face validation demonstrates the degree of resemblance between a method under investigation and real activity. The content validity is established by demonstrating that the system or method measures what it is intended to measure in terms of e.g. surgery or planning [15-17].

Temporal bone (TB) and skull base anatomy are considered among the most complex anatomical regions in humans and their accurate evaluation and understanding is challenging even for experienced otologic and skull base surgeons. The aim of this study was to examine the accuracy of VR compared to cross-sectional viewing and to establish its feasibility in a simulated preoperative planning setting. Our hypothesis is that the accuracy of the VR environment is comparable to cross-sectional PACS viewing: however, it can help providing more accessible information on the topographical anatomy in TBs with better subjective validity compared to cross-sectional viewing.

\section{Materials and methods}

The study had an institutional approval (No. 125/2019) and the National Supervisory Authority for Welfare and Health authorized the use of cadaveric TBs (No. 9202/06.01.03.01/2013). The study fulfilled the Helsinki Declaration for Ethical use of human material.

Five anatomically normal TBs were harvested, and five 3-mm titanium fiducial marker screws were placed on each TB to predefined locations: two at the squamous part, one at the mastoid tip and two at the petrous part (Fig. 1a). Direct physical measurement (DPM) of the distances between screw fiducials were obtained with a standard Vernier caliper (accuracy $0.02 \mathrm{~mm}$ ) under an operating microscope by one expert otologic surgeon. DPMs were later compared with the distance measurements performed in the VR and PACS interface. In addition, 11 measurements of surgically significant anatomical structures (Table 1) such as the size of facial recess and the diameters of the oval and round windows were obtained for every TB in the VR environment and with PACS interface (Figs. 1b, $c$ and $2 b$ ). Since DPMs for anatomical structures were not available, the measurements were compared as a percentage difference to the respective median of the anatomical measurements conducted by the five subjects.

High resolution CT (HRCT) images were acquired using Siemens SOMATOM Definition Flash (Siemens Healthcare, Forchheim, Germany). The imaging parameters for the scans were $120 \mathrm{kV}, 96 \mathrm{mAs}$, FOV $85 \mathrm{~mm}$, pitch 0.8 , CTDIvol $21.1 \mathrm{mGy}$ and slice thickness of $0.4 \mathrm{~mm}$. An experienced neuroradiologist and two experienced surgeons evaluated the quality of the HRCT images both in the PACS interface with conventional cross-sectional viewing (PACS) and in the VR environment (VR).

The Adesante SurgeryVision ${ }^{\mathrm{TM}}$ (Adesante Oy, Turku, Finland) medical software was used to create the VR environment and the 3D model from the HRCT data. A Headset (HTC Vive Pro, HTC, New Taipei, Taiwan) and a pair of controllers were used to interact and visualize the models in the VR environment created by the software (Figs. 1d and 2a). The cross-sectional HRCT image visualization was performed in the PACS interface (Sectra AB, Linkoping, Sweden). 

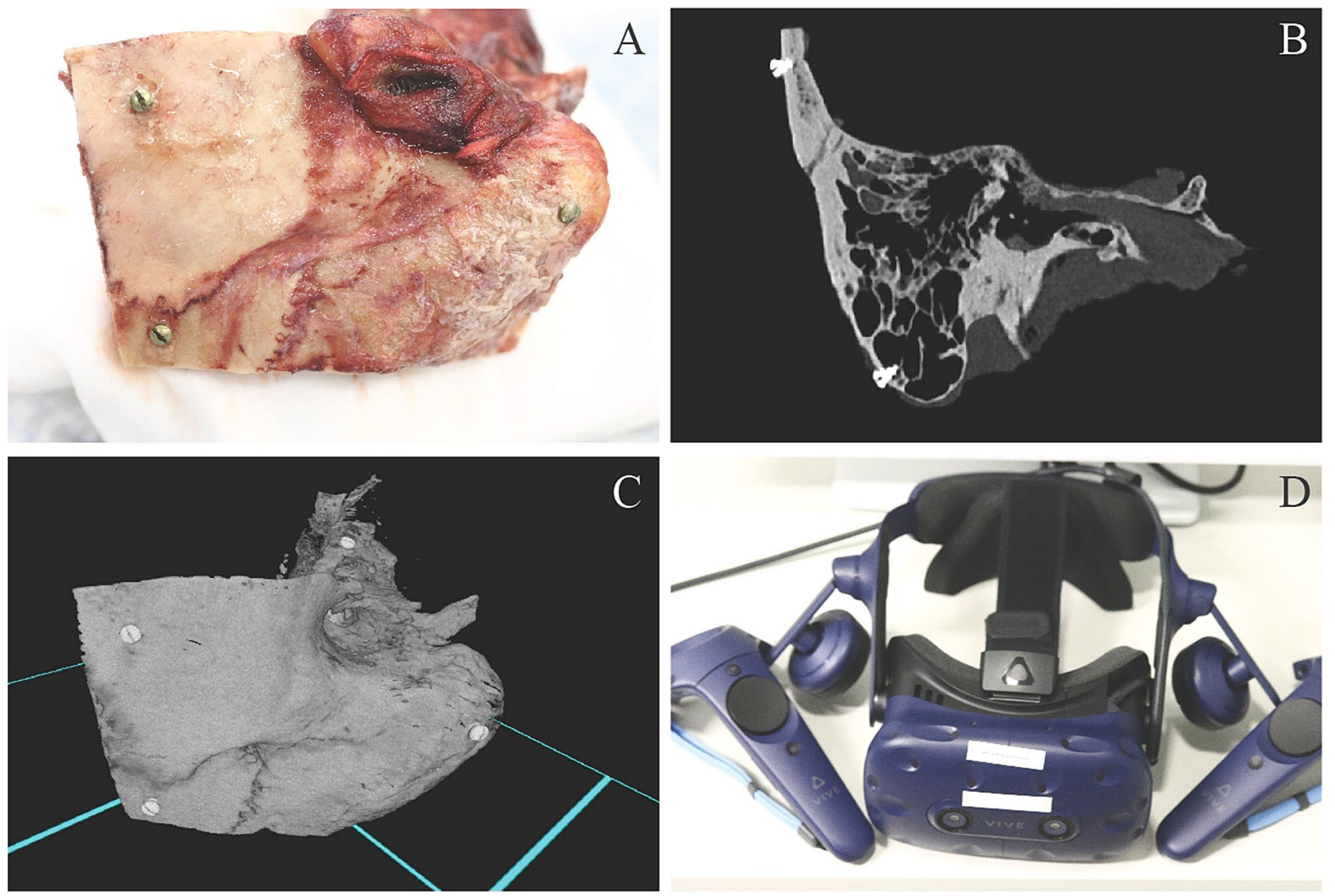

Fig. 1 a Fresh frozen temporal bone, b Conventional 2D HRCT image of temporal bone, c Temporal bone in VR environment, $\mathbf{d}$ HTC Vive Pro head-mounted display and a pair of controllers

Table 1 Measured anatomical distances and their clinical relevance

\begin{tabular}{|c|c|}
\hline Anatomical measurements & Indication for measured distance: \\
\hline Length of malleus (manubrium + head) & Universal measurement, easy to compare, no direct surgical relevance \\
\hline Distance from body of incus to mastoid cortex & The estimate for drilling depth, e.g. in mastoidectomy \\
\hline Horizontal diameter of bony ear canal (external meatus) & Estimation of drilling work during canaloplasty \\
\hline Vertical diameter of bony ear canal (internal meatus) & $\begin{array}{l}\text { Estimation of tympanic membrane diameter, universal anatomic meas- } \\
\text { urement }\end{array}$ \\
\hline Size of facial recess & Space for posterior tympanotomy \\
\hline Distance from facial recess to mastoid cortex & The estimate for drilling depth regarding the facial nerve \\
\hline Distance from facial nerve (mastoid part) to recess of bony ear canal & Limit of posterior drilling in canaloplasty \\
\hline Diameter of oval window & $\begin{array}{l}\text { Stapedotomy, stapedectomy. Identification of the oval window area and } \\
\text { surgical access to stapes footplate }\end{array}$ \\
\hline Diameter of round window & $\begin{array}{l}\text { Cochlear implantation and active middle ear implantation. Identification } \\
\text { of round window area and evaluation of surgical approach }\end{array}$ \\
\hline Length of styloid process & Universal anatomical measurement, stylalgia \\
\hline Distance from sigmoid sinus to back wall of bony ear canal & The space available for drilling in e.g. mastoidectomy \\
\hline
\end{tabular}

\section{Subjects}

Five experienced otologic surgeons volunteered for the study. The mean for working years in the field of ENT was 16.5 years (range 5.5-34 years). The mean for otological operations performed per year was 72 cases (range 48-120). The three less experienced subjects had performed $396-720$ and the two more experienced subjects $1632-2520$ 

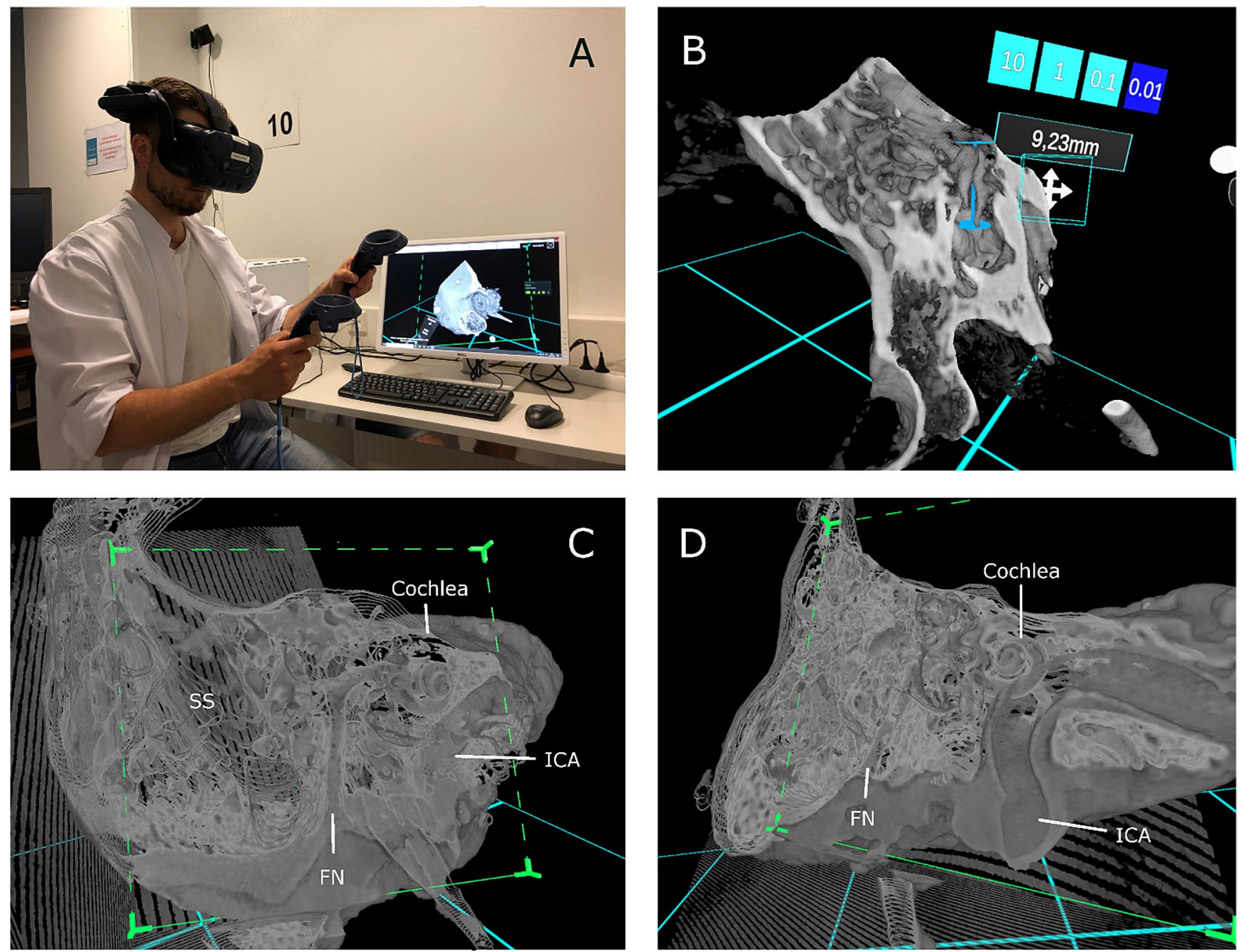

Fig. 2 a VR interface during the measurement of temporal bone, $\mathbf{b}$ measurement of malleus in VR environment, $\mathbf{c}$ temporal bone in VR environment, $\mathbf{d}$ temporal bone in VR environment. SS Sigmoid sinus, FN Facial nerve, ICA Internal carotid artery

otological operations as a primary surgeon. None of the subjects had any prior experience with VR.

\section{Tasks}

Each subject received a 15 -min hands-on instruction for using the VR software. The measurement tasks (see Online Resource 1.) of the fiducials and anatomical landmarks were performed in a predetermined sequence. Three preset windows were optimized to the VR for bone, soft tissue and a translucent bone visualization. In addition, the subjects were allowed to control and adjust the settings as they preferred. All five TBs were measured in randomized order. The corresponding measurements in the PACS interface were performed after a minimum of 2 weeks' interval (mean 175 days) and in random order. Linked multiplanar $2 \mathrm{D}$ reconstructions which display the axial, sagittal and coronal view were used to make the measurements with PACS interface. To obtain the optimal image sections for measurement, subjects were able to rotate the view and planes in any direction (Fig. 2c, d).

\section{Questionnaire}

After completing the tasks, the subjects evaluated the subjective validity of both the methods using a modified fivepoint Likert-type survey (see Online Resource 2.). Similar surveys have been previously used in temporal bone simulator studies [18-20]. The questionnaire included 20 domains in which one represented not true/realistic/useful and five represented very true/realistic/useful. A score of 3 was considered neutral. A free text section was added for specific comments on the perceived advantages and problems. The questionnaire was divided into face (FV) and content validity (CV) and global rating (GR) subgroups assessing user experience, the quality and veracity of the image illustration 
and hardware configuration between the methods, the level of surgical planning effectiveness and anatomy learning, and the applicability of the methods into clinical use, respectively.

\section{Statistical analysis}

The statistical analysis was performed under the consultation of a statistician. All the statistic tests were performed with IBM SPSS statistics version 25 (IBM SPSS, SPSS Inc., Chicago, IL, USA). The level of significance was set to $p<0.05$ for all statistical methods used in this study.

Intraclass correlation coefficient (ICC) was used as a measure of correlation between the distances measured with different methods, i.e. between VR, PACS and DPM. The inter-rater reliability describing the agreement between different subjects was defined separately for VR and PACS measurements of fiducial and anatomical distances. The anatomical distance measurements were converted and evaluated as a percentage difference compared to the median of each measurement point.

The two-tailed paired sample $t$-test and Bland-Altman analysis were performed to evaluate the equivalence of the PACS and VR measurements [21]. A linear mixed model was used to consider the possible measurement bias between different subjects. For box plots, the measurements 1.5 IQRs above the upper and below the lower quartiles were determined as outliers. Wilcoxon signed rank test was conducted for the Likert-type questionnaire statistical analysis.

\section{Results}

All subjects completed all the given tasks. Due to a partially fractured bony ear canal in one TB eleven anatomical measures $(11 / 275,4 \%)$ could not be determined. The missing measurements were not method dependable (PACS data $6 / 11$, VR data 5/11). A summary of all measurements and statistics are presented in Tables 2 and 3 .

The box plots illustrating the distribution of the measurements are presented in Fig. 3. The statistical data outliers were interpreted as measurement errors. Every subject made measurement errors with both methods. The total number of errors in the anatomical measurements were 58 in VR data and 27 in PACS data (Fig. 3b). The errors in the fiducial measurements were more frequent (26 vs 5) (Fig. 3a) and the mean difference to DPM was higher in PACS data than in VR data (Table 2). Of the 26 errors made in the cross-sectional viewing, 14 (54\%) were most likely due to a misidentification of the fiducial measurement points (measured distance matched to a distance between other, not aimed, fiducial pair). None of the measurement errors in VR suited for fiducial misidentification. Subject 4 made several incorrect identifications of the fiducials (seven identification mistakes) in the cross-sectional viewing leading to substantial measurement errors (13 data point outliers in total), but misidentifications were also made by the more experienced subjects. There was no difference in the number of measurement errors in total

Table 2 Statistics in screw fiducial distance measurements obtained in conventional cross-sectional view (PACS) and in the VR environment

\begin{tabular}{|c|c|c|c|c|c|c|c|c|}
\hline Subject & Method & $\begin{array}{l}\text { Mean differ- } \\
\text { ence to DPM } \\
{[\mathrm{mm}]}\end{array}$ & $\mathrm{SD}[\mathrm{mm}]$ & $\begin{array}{l}95 \% \mathrm{CI} \text { for mean } \\
\text { difference }[\mathrm{mm}]\end{array}$ & $\begin{array}{l}\text { Mean difference } \\
\text { between PACS } \\
\text { and VR }[\mathrm{mm}]^{\mathrm{a}}\end{array}$ & $\begin{array}{l}\text { ICC PACS vs. } \\
\text { VR }\end{array}$ & $\begin{array}{l}\text { ICC PACS vs. } \\
\text { DPM }\end{array}$ & ICC VR vs. DPM \\
\hline \multirow[t]{2}{*}{1} & PACS & -0.291 & 1.344 & $-0,673 ; 0.091$ & \multirow{2}{*}{$\begin{array}{l}-0.076 \\
\quad(p=0.688)\end{array}$} & \multirow[t]{2}{*}{0.995} & \multirow[t]{2}{*}{0.995} & \multirow[t]{2}{*}{0.998} \\
\hline & VR & -0.215 & 0.986 & $-0.495 ; 0.065$ & & & & \\
\hline \multirow[t]{2}{*}{2} & PACS & -0.181 & 3.187 & $-1.087 ; 0.724$ & \multirow{2}{*}{$\begin{array}{l}-0.010 \\
\quad(p=0.982)\end{array}$} & \multirow[t]{2}{*}{0.973} & \multirow[t]{2}{*}{0.974} & \multirow[t]{2}{*}{0.998} \\
\hline & VR & -0.171 & 0.895 & $-0.425 ; 0.083$ & & & & \\
\hline \multirow[t]{2}{*}{3} & PACS & -1.129 & 5.295 & $-2.634 ; 0.375$ & \multirow{2}{*}{$\begin{array}{l}-0.565 \\
\quad(p=0.442)\end{array}$} & \multirow[t]{2}{*}{0.933} & \multirow[t]{2}{*}{0.929} & \multirow[t]{2}{*}{0.998} \\
\hline & VR & -0.565 & 0.852 & $-0.807 ;-0.323$ & & & & \\
\hline \multirow[t]{2}{*}{4} & PACS & 2.563 & 5.524 & $0.993 ; 4.133$ & \multirow{2}{*}{$3.371\left(p<0.001^{*}\right)$} & \multirow[t]{2}{*}{0.927} & \multirow[t]{2}{*}{0.916} & \multirow[t]{2}{*}{0.997} \\
\hline & VR & -0.808 & 1.066 & $-1.111 ;-0.505$ & & & & \\
\hline \multirow[t]{2}{*}{5} & PACS & -0.591 & 1.198 & $-0.932 ;-0.251$ & \multirow{2}{*}{$\begin{array}{l}-0.361 \\
\quad\left(p=0.005^{*}\right)\end{array}$} & \multirow[t]{2}{*}{0.998} & \multirow[t]{2}{*}{0.996} & \multirow[t]{2}{*}{0.998} \\
\hline & VR & -0.230 & 0.938 & $-0.497 ; 0.036$ & & & & \\
\hline \multirow[t]{2}{*}{$\operatorname{Total}^{\mathrm{b}}$} & PACS & 1.753 & 3.563 & $1.178 ; 2.327$ & \multirow[t]{2}{*}{$0.472(p=0.065)$} & \multirow[t]{2}{*}{0.965 (mean) } & \multirow[t]{2}{*}{0.962 (mean) } & \multirow[t]{2}{*}{0.998 (mean) } \\
\hline & VR & 0.815 & 0.665 & $0.707 ; 0.922$ & & & & \\
\hline
\end{tabular}

$S D$ standard deviation, $C I$ confidence interval, ICC intraclass correlation coefficient $(p<0.001), P A C S$ conventional cross-sectional (2D) method, $V R$ virtual reality environment, $D P M$ direct physical measurements by Vernier caliper

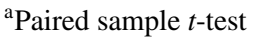

${ }^{\mathrm{b}}$ Absolute measurement values used

*Statistically significant difference between the methods 
Table 3 Statistics in anatomical distance measurements obtained in the conventional cross-sectional view (PACS) and in the VR environment

\begin{tabular}{|c|c|c|c|c|c|c|}
\hline Subject & Method & Mean difference [\%] & $\mathrm{SD}[\%]$ & $\begin{array}{l}95 \% \text { CI for mean dif- } \\
\text { ference }[\%]\end{array}$ & $\begin{array}{l}\text { Mean difference between } \\
\text { PACS and VR [\%] }{ }^{\mathrm{a}}\end{array}$ & ICC PACS vs. VR \\
\hline \multirow[t]{2}{*}{1} & PACS & -0.198 & 11.791 & $-3.386 ; 2.989$ & $2.825(p=0.186)$ & 0.978 \\
\hline & VR & -3.023 & 16.621 & $-7.517 ; 1.470$ & & \\
\hline \multirow[t]{2}{*}{2} & PACS & 4.130 & 18.650 & $-0.912 ; 9.172$ & $1.980(p=0.519)$ & 0.923 \\
\hline & VR & 2.113 & 16.075 & $-2.232 ; 6.495$ & & \\
\hline \multirow[t]{2}{*}{3} & PACS & -3.097 & 22.239 & $-9.109 ; 2.915$ & $-4.864(p=0.174)$ & 0.936 \\
\hline & VR & 1.915 & 20.863 & $-3.690 ; 7.591$ & & \\
\hline \multirow[t]{2}{*}{4} & PACS & -2.988 & 59.020 & $-18.943 ; 12.967$ & $11.054(p=0.268)$ & 0.900 \\
\hline & VR & -14.137 & 39.732 & $-24.878 ;-3.396$ & & \\
\hline \multirow[t]{2}{*}{5} & PACS & 2.645 & 16.863 & $-1.914 ; 7.203$ & $0.300(p=0.971)$ & 0.971 \\
\hline & VR & 2.388 & 13.900 & $-1.379 ; 6.146$ & & \\
\hline \multirow[t]{2}{*}{ Total $^{\mathrm{b}}$} & PACS & 12.824 & 27.451 & $9.565 ; 16.083$ & $1.923(p=0.396)$ & 0.942 (mean) \\
\hline & VR & 13.140 & 20.238 & $10.737 ; 15.542$ & & \\
\hline
\end{tabular}

Measurements evaluated as a percentage difference compared to the median.

$S D$ standard deviation, $C I$ confidence interval, ICC intraclass correlation coefficient $(p<0.001), P A C S$ conventional cross-sectional (2D) method, $V R$ virtual reality environment

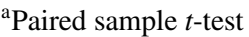

${ }^{\mathrm{b}}$ Absolute measurement values used

Fig. 3 Box plots for the comparison of a fiducial (percentile error compared to DPMs) and b anatomical (percentile error compared to median of each anatomical measurement point) measurement accuracy from all subjects
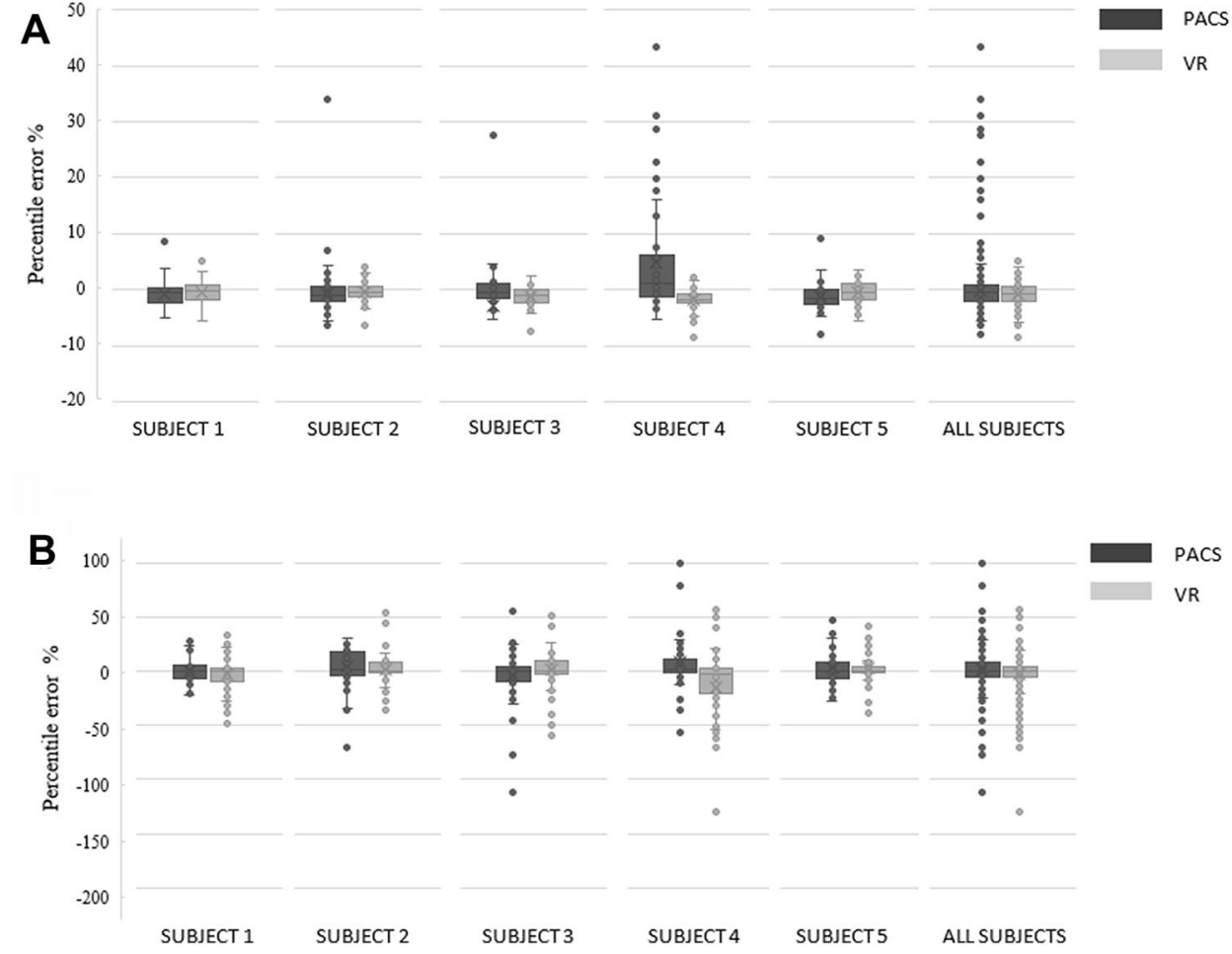

between less or more experienced otologic surgeons (mean 23.7 vs. 23.0 outliers) or between methods used by less or more experienced subjects (PACS data 10.3 vs. 11.0, VR data 13.3 vs. 12.0), respectively. Thus, in this study the level of surgical experience appeared not to have an impact on the number of measurement errors in either method.
A strong correlation between the methods was found for the measurements of the fiducial (ICC $\geq 0.916$; Table 2) and of the anatomical distances (ICC $\geq 0.900$; Table 3). The inter-rater reliability was high in both PACS and VR methods: in the fiducial distance measurements, the mean ICC between the subjects was 0.930 and 0.999 for PACS 
data and VR data, respectively. Accordingly, in the anatomical distance measurements, the respective mean ICCs were 0.914 and 0.955 for PACS data and VR data.

There were no statistically significant differences in the fiducial $(p=0.065)$ and anatomical landmark measurements $(p=0.396)$ between PACS and VR data (Tables 2 and 3). However, when performing the paired sample t-test separately for each subject, we found a significant difference between the methods in two subjects in the fiducial distance measurements (Table 2). Subject 4 made significant mistakes in identification of the fiducials in the PACS interface, which explains this finding. In the contrary, the excellent measurement agreement of subject 5 resulted in a very low standard deviation and narrow confidence interval (Fig. 3, Table 2), which explains the statistical significance.

The Bland-Altman plots are presented in Fig. 4. The differences for PACS and VR data in both anatomical and fiducial measurements fell mainly within the limits of agreement for every subject. There was no systematic bias between the two methods.

All subjects considered VR helpful for the assessment of TB anatomy. Mean overall Likert scores for VR were significantly better compared to PACS scores $(p<0.001)$. In subgroup analysis, Likert scores were significantly better towards the VR for FV $(p=0.002)$ and CV $(p<0.001)$. The GR subgroup questions showed a tendency in favor of the VR method, but the difference remained statistically insignificant $(p=0.132)$ (Table 4$)$. The analysis of the individual questions of the survey revealed statistically significant differences between the two methods presented in Table 5. The appearance of the anatomical structures, the depth perception, the understanding of the anatomical structures (e.g. the cochlea rotation within the labyrinth), the hand-eye coordination (e.g. how easy it is to grasp, move and follow the objects) and overall score for the understanding of the surgical site (e.g. in which angle to approach to round window or the risk for facial nerve damage in posterior tympanomy) in VR were significantly preferred over PACS. The mean scores for ergonomics (3.2 vs 3.6) and the subjects grade for feasibility and inclusion of the method to clinical surgical planning use (4.6 vs 4.8) were the only questions that were in favor of PACS but statistical significance ( $p=0.317)$ could not be presented.

The analysis of the free text feedback from the questionnaire indicated that VR gave better 3D understanding of the anatomical structures and their relationship. The possibility to approach the target in any angle and direction was appreciated. All subjects valued the VR as an excellent tool for teaching anatomy and for training otologic surgery. None of the subjects reported VR to cause vertigo, nausea or headache.

\section{Discussion}

Several studies, especially in reconstructive surgery, have demonstrated, that virtual surgical planning may improve surgical outcomes and accuracy [22, 23]. For TB anatomy specifically, few studies have evaluated virtual temporal bone dissection software for training purposes [24-26]. In a case-specific study of VR temporal bone surgery simulator $75 \%$ of users, comprising trainers and trainees, valuated VR useful for preoperative planning [27]. To the best of our knowledge, there are no clinical studies investigating the measurement accuracy of VR for TB anatomy and simulated surgical planning with expert otosurgeons. In the present study, we explored the feasibility, measurement accuracy and the subjective validity of VR planning in simulated setting with TBs and compared it to the planning in the PACS interface with standard cross-sectional viewing.

The five test subjects were experienced otologic surgeons of a tertiary center with an expert-level knowledge of temporal bone anatomy and surgical planning in the PACS interface but no prior experience with consumer or medical VR environments. The results support our hypothesis with respect to the feasibility and measurement accuracy of the used VR software of complex anatomic models such as TBs compared to cross-sectional viewing. More specifically, we found strong correlations and agreement between all distance measurements for each method (Tables 2 and 3; Fig. 4). In fact, the distance measurements in the VR environment correlated stronger with the DPM compared to those obtained in PACS interface. Similar strong correlations between VR and corresponding physical measurements have been established with previous study [13].

Since the DPMs were not available for the anatomical measurements only the correlation between both methods could be investigated. The variation in the anatomical measurements were higher in the VR than in the crosssectional viewing (Table 3; Fig. 3b) but both methods demonstrated strong correlation.

Interestingly, in the cross-sectional viewing 14 fiducial distances were misidentified by the subjects, whereas no identification errors were made in the VR. This also contributed to the lower standard deviation for the VR measurements (Table 2), which, however, remained statistically insignificant. In addition, the measurements of anatomical landmark distances were comparable between the methods. This finding is rather compelling considering its possible clinical implications for surgical planning. It suggests that VR environment may offer more measurement accuracy of complex anatomical structures with less measurement and identification errors even for experienced surgeons. 


\section{Bland Altman Plots}

\section{Anatomical measures}

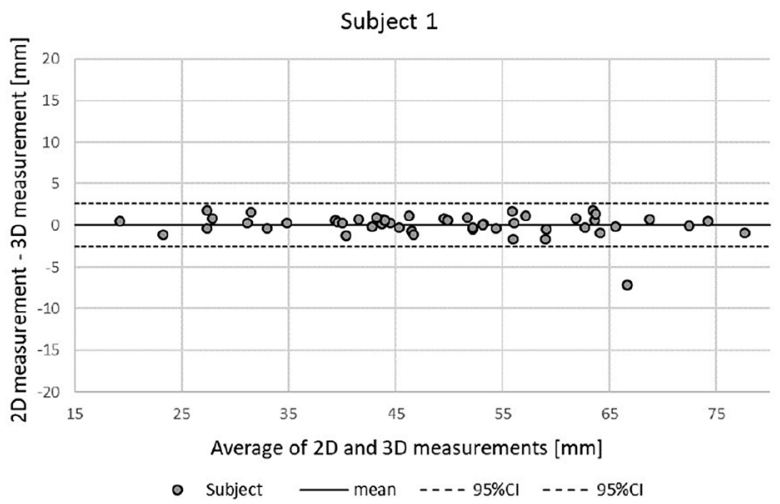

Subject 2

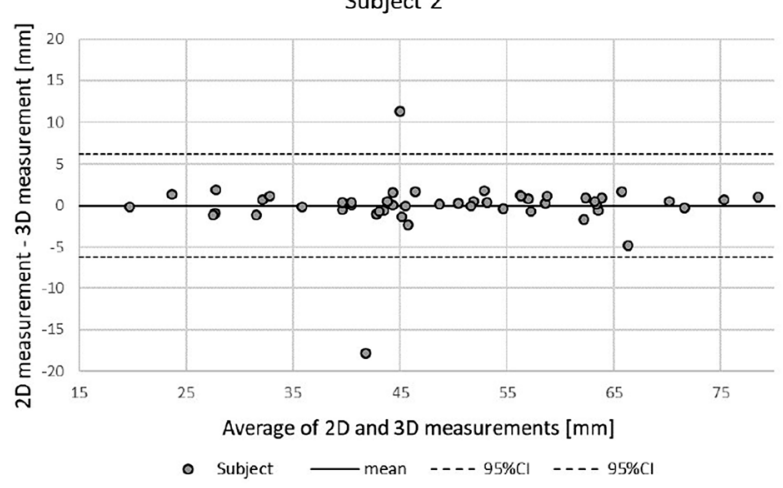

Subject 3

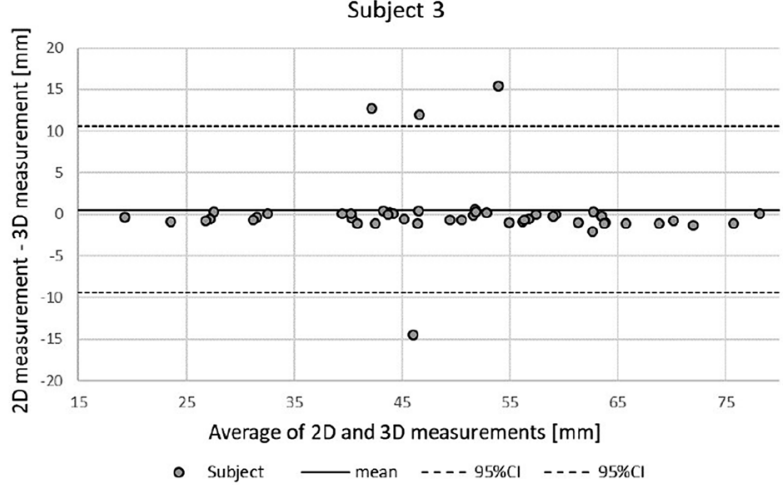

Fiducial measures

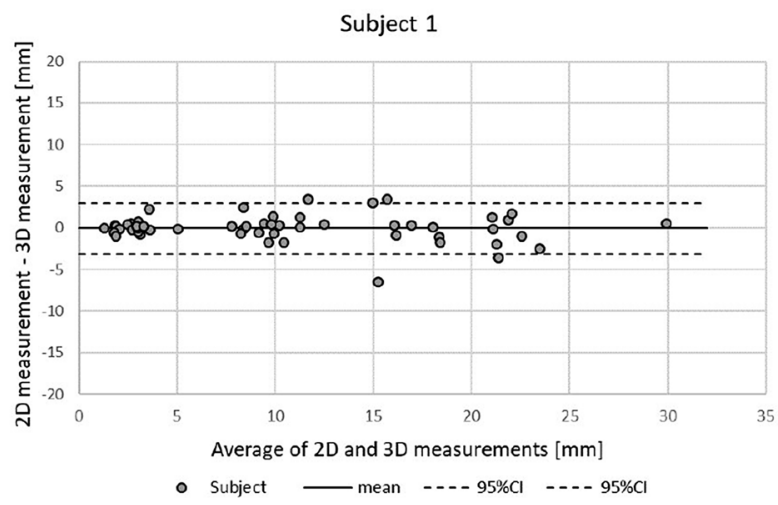

Subject 2

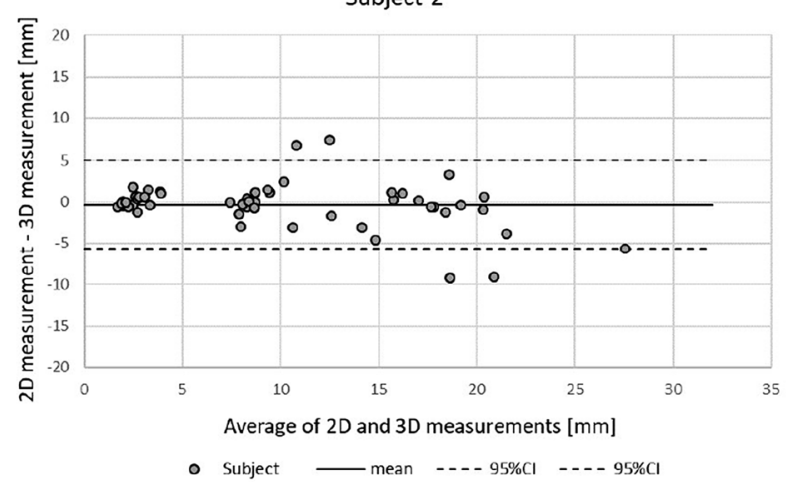

Subject 3

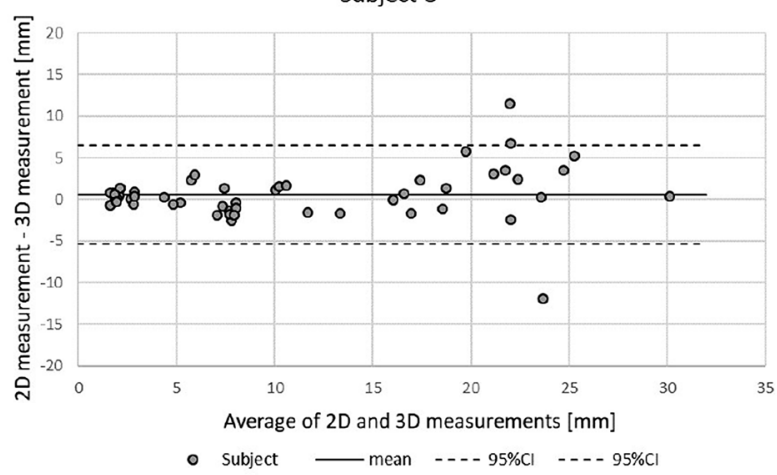

Fig. 4 Bland-Altman plots for the comparison of anatomical measurements and fiducial measurements from all subjects

This is in accordance to previous clinical studies mainly done in reconstructive surgery, which have shown that VR planning improve the accuracy of the surgical outcomes $[22,23,28-31]$.

All subjects favored VR over cross-sectional viewing in PACS interface for a more detailed apprehension of anatomy and topography. VR was also regarded beneficial for learning complex anatomy and surgical planning. VR environment allowed more detailed definition and measurements of surgically relevant spaces and distances compared to cross-sectional viewing. For example, the size of facial recess, diameter of round window were found much more assessable in VR environment. In addition, topographical relations can be better perceived in the VR environment 


\section{Bland Altman Plots}

\section{Anatomical measures}

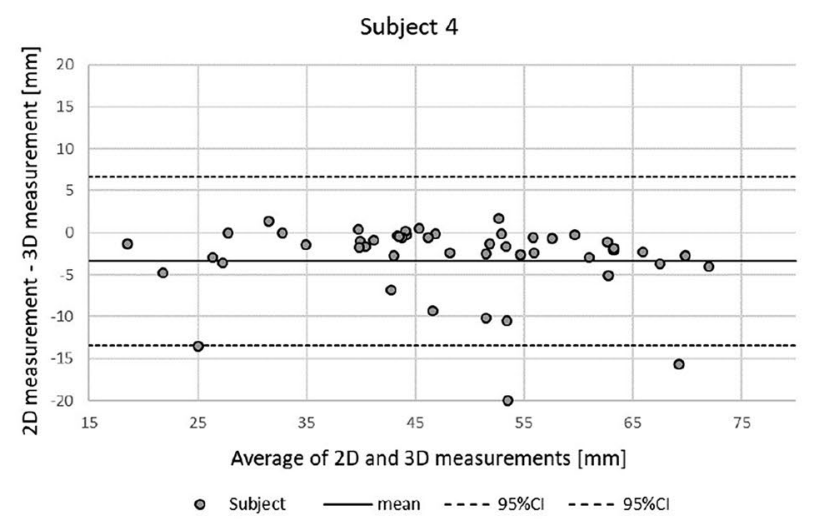

Subject 5

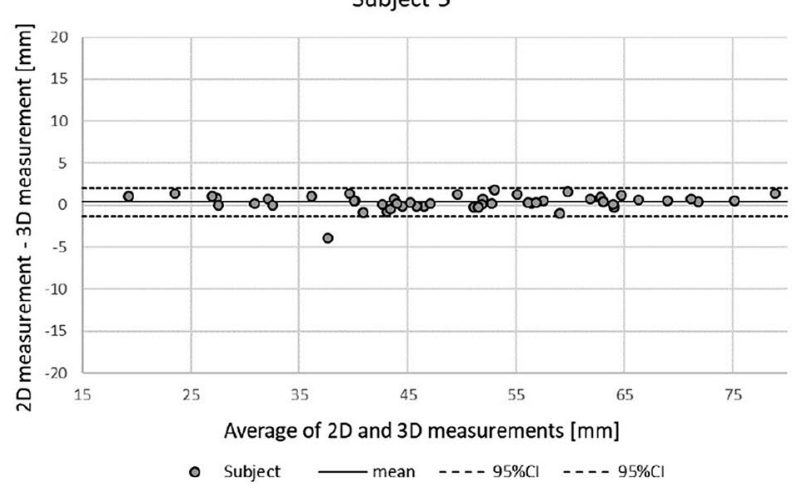

Fig. 4 (continued)

Table 4 Wilcoxon signed rank test for the 5-point Likert questionnaire

\begin{tabular}{llllll}
\hline $\begin{array}{l}\text { Question- } \\
\text { naire } \\
\text { subgroup }\end{array}$ & Method & Mean score & SD & Diff. (95\% CI) & $p$ value \\
\hline FV & PACS & 3.20 & 0.99 & $0.58(-1.00 ;$ & $0.002^{*}$ \\
& VR & 3.78 & 0.83 & $0.00)$ & \\
CV & PACS & 3.23 & 0.62 & $0.9(-1.50 ;$ & $<0.001^{*}$ \\
& VR & 4.33 & 0.62 & $1.00)$ & \\
GR & PACS & 4.10 & 0.85 & $0.25(-0.50$ & 0.132 \\
& VR & 4.35 & 0.75 & $; 0.00)$ & \\
Total & PACS & 3.39 & 0.90 & $0.72(0.50 ;$ & $<0.001^{*}$ \\
& VR & 4.11 & 0.78 & $1.00)$ & \\
\end{tabular}

$F V$ Face validation, $C V$ Content validation, $G R$ Global rating, $S D$ standard deviation, $V R$ virtual reality environment, PACS conventional cross-sectional (2D) method

*Statistically significant difference between the methods

\section{Fiducial measures}
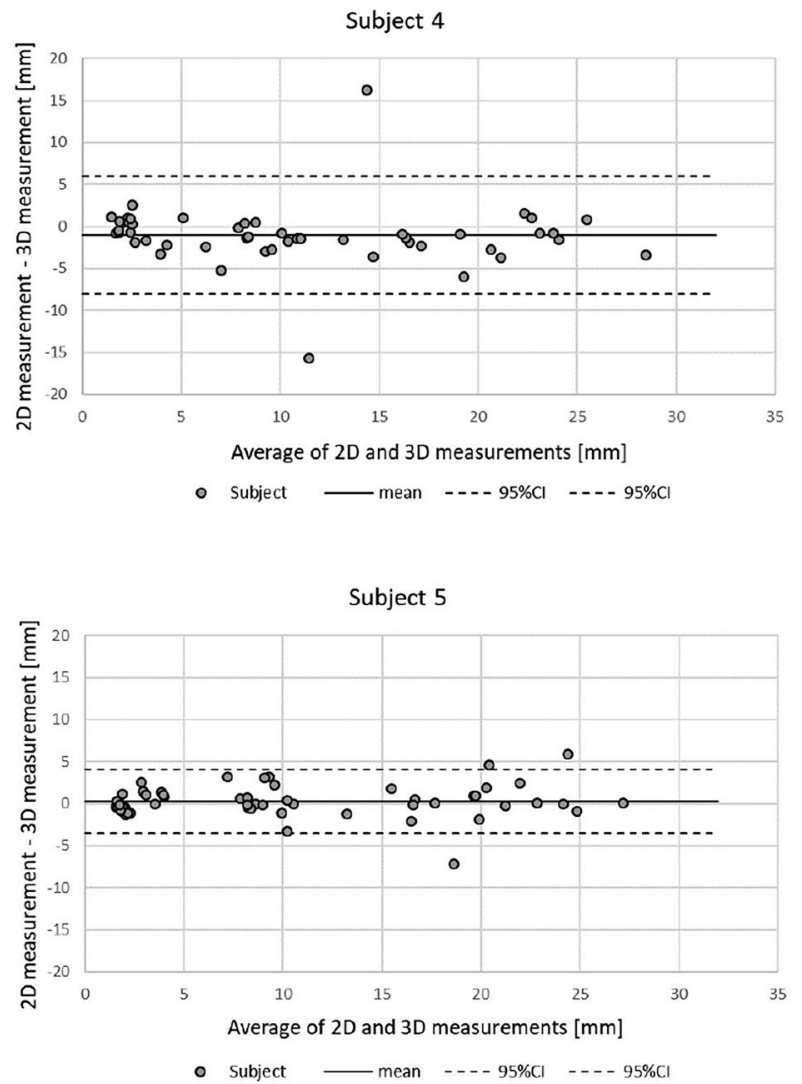

which clinically can help to conceive, e.g. the insertion trajectory of a cochlear implant electrode.

The measurement tasks were also reported to be easier with the VR (Fig. 3). The subjects commonly reported that the accurate identification of the fiducials and the related measurements were difficult in the cross-sectional viewing. Especially, the alignment of the fiducials was considered more difficult, although the objective to use fiducial markers was to obtain fixed measurement points to mitigate the marginal for measurement errors. As mentioned earlier, all experienced surgeons misidentified some fiducials more frequently in PACS interface than in VR. This underlines our main finding that the VR environment provides more accessible information in complex anatomic situations, which is in accordance to previous studies [32-34]. There are significant differences in spatial comprehension between individuals, which affect their ability to perform an anatomical task [35, 36]. Surgeons too, including also the most experienced, are subject to the same variations in the ability to reconstruct 
Table 5 Wilcoxon signed rank test for individual questions of the 5-point Likert questionnaire

\begin{tabular}{llll}
\hline & VR (SD) & PACS (SD) & $p$ value \\
\hline FV subgroup & & & \\
Appearance of anatomical structures & $4.6(0.5)$ & $3.4(0.5)$ & $0.034^{*}$ \\
Appearance of tools & $3.2(0.4)$ & $3.2(0.7)$ & 1.000 \\
Usability of tools & $3.0(0.6)$ & $3.0(1.1)$ & 1.000 \\
Performance of tools & $3.8(0.4)$ & $3.4(0.5)$ & 0.157 \\
Haptic feedback & $3.6(0.5)$ & $3.0(0.6)$ & 0.830 \\
Ergonomics & $3.2(0.4)$ & $3.6(1.0)$ & 0.317 \\
Depth perception & $4.4(0.8)$ & $2.0(0.9)$ & $0.034^{*}$ \\
Quality of graphics & $4.4(0.8)$ & $4.0(0.9)$ & 0.317 \\
CV subgroup & & & \\
Learning of anatomy & $4.6(0.5)$ & $3.2(0.4)$ & 0.059 \\
Learning of surgical planning & $4.6(0.5)$ & $3.4(0.5)$ & 0.063 \\
Understanding of anatomical structures & $4.4(0.5)$ & $3.4(0.5)$ & $0.034^{*}$ \\
Quality of measuring anatomical structures & $4.4(0.5)$ & $2.8(0.7)$ & 0.059 \\
Understanding the relationships of anatomical & $4.6(0.5)$ & $3.0(0.6)$ & 0.180 \\
$\quad$ structures & & & \\
Accuracy of measurement tool & $4.0(0.9)$ & $3.6(0.8)$ & 0.577 \\
Hand-eye-coordination & $3.8(0.4)$ & $3.0(0.0)$ & $0.046^{*}$ \\
Overall score for surgical planning & $4.2(0.4)$ & $3.4(0.5)$ & 0.102 \\
GR subgroup & & & 0.157 \\
Recommend to colleague & $4.6(0.5)$ & $4.2(0.7)$ & 1.000 \\
User-friendly & $3.6(0.8)$ & $3.6(1.0)$ & 0.317 \\
Inclusion to surgical planning & $4.6(0.5)$ & $4.8(0.4)$ & $0.046^{*}$ \\
Understanding of the surgical site & $4.6(0.5)$ & $3.8(0.4)$ & \\
\hline & & & \\
\hline
\end{tabular}

Subjects $(n=5)$

$V R$ virtual reality environment, PACS conventional cross-sectional (2D) method. $F V$ Face validation, $C V$ Content validation, $G R$ Global rating, $S D$ standard deviation

* Statistically significant difference between the methods volumetric data into a complex three-dimensional model in their mind. This is a challenging process especially for novice surgeons but may mislead also experienced surgeons like our results showed.

Some adverse effects have been previously described with the VR application use [37]. The use of head-mounted displays has been associated with nausea, oculomotor disturbances, disorientation and headache [38, 39]. In this study none of the subjects reported any adverse effects. The limited number of subjects and the diminutive need for quick turns of gaze and head in VR environment possibly caused low sensory mismatch not strong enough to generate these adverse effects.

Several strengths and limitations of this study require attention. The 5-point Likert questionnaire was limited for subjective evaluation, which is a common limitation with self-reported validation surveys. The number of test subjects was limited with different levels of otology and skull base experience, which may have confounded the results. Although not statistically significant, less experience appeared to negatively affect cross-sectional viewing. This is not very surprising since an unambiguous allocation of the fiducials or anatomic structures is easier for the visualization in VR. Since these mistakes occurred also to the most experienced surgeons, it can be doubted whether a larger number of subjects would have changed the present results. The relatively large number of measurements ( 525 per subject) reduced the effect of individual errors and added to the statistical power of this study and could substantiate the objective validity of the VR software for surgical planning.

This study concentrated to test the measurement accuracy of VR environment developed for surgical planning compared to conventional cross-sectional image viewing and distance measurements without temporal dissection or clinical surgery. To test the VR environment in mastoidectomy for evaluation of its feasibility in the clinical setting warrants for further studies. 3D reconstructions could also be visualized on a $2 \mathrm{D}$ monitor. Conventional calibrated high-quality radiological monitors may have better image quality, especially for surface and bone anatomy, and accessibility in hospitals than the current VR systems. However, VR visualization provides better stereoscopicity and immersivity than $3 \mathrm{D}$ 
reconstructions visualized in 2D monitors. The movements in virtual space are more natural and correspond better the surgical reality. Thus, it would be interesting to also compare to the $3 \mathrm{D}$ reconstructions on $2 \mathrm{D}$ monitors, conventional 2D cross-sectional viewing and VR for surgical planning in the future.

The development of more advanced VR environments and augmented reality (AR) environments with haptic devices will have a major impact on surgical planning, training and navigation in the future [40]. The AR allows users to merge both the real and virtual environments and is a continually improving technology of high interest for further studies [41]. The accurate visualization of complex anatomic structures is indispensable for adequate planning. The VR environment allows surgeons to engage the patients imaging studies like the anatomy during the surgery and simulate, e.g. the access or approach for a given patient. As the field of surgery evolves towards more patient specific and mini-invasive techniques, new techniques and tools for preoperative simulation and image viewing is needed. This study demonstrated that the accuracy of the VR environment for the assessment of TB anatomy is at least comparable to conventional cross-sectional viewing, but with the added benefit of providing more comprehensive information on topographical and spatial relation of anatomic structures. For the less experienced, the VR environment may represent an even more important tool for learning complex anatomy. Therefore, it is also important to investigate the usefulness of the VR environment for learning TB anatomy in medical students and novice otologic surgeons.

\section{Conclusion}

The present study demonstrated the feasibility of VR for simulated surgical planning in TBs. The VR environment provided comparable results with less measurement errors than conventional cross-sectional viewing, confirming its feasibility in clinical image viewing for simulated surgical planning and adding an in-depth apprehension of complex anatomy. In addition, VR demonstrated better face and content validity compared to PACS, confirming its subjective validity. Further studies are needed to establish and confirm the objective validity and the effect of the VR surgical planning on clinical surgical outcomes in otology.

Acknowledgements The authors give their thanks to Sini Varonen MD, Antti Ruuskanen MD, Timo Karjalainen and Jyrki Tervaniemi MDD for their participation in this study. We also would like to thank Tuomas Selander MSc., for his assistance in the statistical analyses.

Funding Open access funding provided by University of Eastern Finland (UEF) including Kuopio University Hospital. The study was funded by the Academy of Finland, State Research Funding of the
Kuopio University Hospital, the Finnish ORL-HNS Foundation and the Kuopio University Hospital Research Foundation. The research infrastructure (Microsurgery Centre of Eastern Finland) was supported by the Regional Council of Pohjois-Savo and the European Regional Development funds.

\section{Compliance with ethical standards}

Conflict of interest The authors have no conflict of interest to disclose.

Ethics approval The study had an institutional approval (No. 125/2019) and the National Supervisory Authority for Welfare and Health authorized the use of cadaveric TBs (No. 9202/06.01.03.01/2013). The study fulfilled the Declaration of Helsinki for Ethical use of human material.

Informed consent Informed consent was obtained from all subjects.

Open Access This article is licensed under a Creative Commons Attribution 4.0 International License, which permits use, sharing, adaptation, distribution and reproduction in any medium or format, as long as you give appropriate credit to the original author(s) and the source, provide a link to the Creative Commons licence, and indicate if changes were made. The images or other third party material in this article are included in the article's Creative Commons licence, unless indicated otherwise in a credit line to the material. If material is not included in the article's Creative Commons licence and your intended use is not permitted by statutory regulation or exceeds the permitted use, you will need to obtain permission directly from the copyright holder. To view a copy of this licence, visit http://creativecommons.org/licenses/by/4.0/.

\section{References}

1. Kashikar TS, Kerwin TF, Moberly AC, Wiet GJ (2019) A review of simulation applications in temporal bone surgery. Laryngoscope Investig Otolaryngol 4(4):420-424

2. Kraeima J, Dorgelo B, Gulbitti HA, Steenbakkers RJHM, Schepman KP, Roodenburg JLN et al (2018) Multi-modality 3D mandibular resection planning in head and neck cancer using CT and MRI data fusion: a clinical series. Oral Oncol 81:22-28

3. Francis HW, Malik MU, Diaz Voss Varela DA, Barffour MA, Chien WW, Carey JP et al (2012) Technical skills improve after practice on virtual-reality temporal bone simulator. Laryngoscope. 122(6):1385-1391

4. Lee C, Wong GKC (2019) Virtual reality and augmented reality in the management of intracranial tumors: a review. J Clin Neurosci 62:14-20

5. Chinnock C (1994) Virtual reality in surgery and medicine. Hosp Technol 13(18):1

6. Triepels CPR, Smeets CFA, Notten KJB, Kruitwagen RFPM, Futterer JJ, Vergeldt TFM et al (2020) Does three-dimensional anatomy improve student understanding? Clin Anat 33(1):25

7. Krokos E, Plaisant C, Varshney A (2019) Virtual memory palaces: immersion aids recall. Virtual Real 23(1):1-15

8. Kennedy RS, Lane NE, Berbaum KS, Lilienthal MG (1993) Simulator sickness questionnaire: an enhanced method for quantifying simulator sickness. Int J Aviat Psychol 3(3):203-220

9. Rebenitsch L, Owen C (2016) Review on cybersickness in applications and visual displays. Virtual Real 20(2):101-125

10. Rose AS, Kim H, Fuchs H, Frahm JM (2019) Development of augmented-reality applications in otolaryngology-head and 
neck surgery. Laryngoscope 129(Suppl 3):S1-S11. https://doi. org/10.1002/lary.28098

11. Rodby KA, Turin S, Jacobs RJ, Cruz JF, Hassid VJ, Kolokythas A et al (2014) Advances in oncologic head and neck reconstruction: systematic review and future considerations of virtual surgical planning and computer aided design/computer aided modeling. $\mathbf{J}$ Plast Reconstr Aesthet Surg 67(9):1171-1185

12. Witjes MJH, Schepers RH, Kraeima J (2018) Impact of 3D virtual planning on reconstruction of mandibular and maxillary surgical defects in head and neck oncology. Curr Opin Otolaryngol Head Neck Surg 26(2):108-114

13. Xavier B, Hansmann J, Ansong E, Chen J, Zhao L, Michals E (2019) Accuracy and reproducibility of linear and angular measurements in virtual reality: a validation study. J Digital Imaging 33(1):111-120. https://doi.org/10.1007/s10278-019-00259-3

14. Van Nortwick SS, Lendvay TS, Jensen AR, Wright AS, Horvath KD, Kim S (2010) Methodologies for establishing validity in surgical simulation studies. Surgery. 147(5):622-630

15. Fried G, Feldman L (2008) Objective assessment of technical performance. World J Surg 32(2):156-160

16. Carter FJ, Schijven MP, Aggarwal R, Grantcharov T, Francis NK, Hanna GB et al (2006) Consensus guidelines for validation of virtual reality surgical simulators. Simul Healthc 1(3):171-179

17. Mick PT, Arnoldner C, Mainprize JG, Symons SP, Chen JM (2013) Face validity study of an artificial temporal bone for simulation surgery. Otol Neurotol 34(7):1305-1310

18. Varoquier M, Hoffmann CP, Perrenot C, Tran N, Parietti-Winkler C (2017) Construct, face, and content validation on Voxel$\mathrm{Man}(\mathrm{R})$ simulator for otologic surgical training. Int J Otolaryngol 2017:2707690

19. Compton EC, Agrawal SK, Ladak HM, Chan S, Hoy M, Nakoneshny SC et al (2020) Assessment of a virtual reality temporal bone surgical simulator: a national face and content validity study. J Otolaryngol Head Neck Surg 49(1):17

20. Arora A, Khemani S, Tolley N, Singh A, Budge J, Diaz Voss Varela DA et al (2012) Face and content validation of a virtual reality temporal bone simulator. Otolaryngol Head Neck Surg 146(3):497-503

21. Bland JM, Altman DG (1999) Measuring agreement in method comparison studies. Stat Methods Med Res 8(2):135-160

22. Wilde F, Hanken H, Probst F, Schramm A, Heiland M, Cornelius C (2015) Multicenter study on the use of patient-specific CAD/ CAM reconstruction plates for mandibular reconstruction. Int $\mathrm{J}$ CARS 10(12):2035-2051

23. Bell RB (2010) Computer planning and intraoperative navigation in cranio-maxillofacial surgery. Oral Maxillofac Surg Clin North Am 22(1):135-156

24. Locketz GD, Lui JT, Chan S, Salisbury K, Dort JC, Youngblood $P$ et al (2017) Anatomy-specific virtual reality simulation in temporal bone dissection: perceived utility and impact on surgeon confidence. Otolaryngol Head Neck Surg 156(6):1142-1149

25. Frithioff A, Sørensen M, Andersen S (2018) European status on temporal bone training: a questionnaire study. Eur Arch Otorhinolaryngol 275(2):357-363

26. Chan S, Li P, Locketz G, Salisbury K, Blevins NH (2016) Highfidelity haptic and visual rendering for patient-specific simulation of temporal bone surgery. Comput Assist Surg 21(1):85-101
27. Arora A, Swords C, Khemani S, Awad Z, Darzi A, Singh A et al (2014) Virtual reality case-specific rehearsal in temporal bone surgery: a preliminary evaluation. Int J of Surg 12(2):141-145

28. Shenaq DS, Matros E (2018) Virtual planning and navigational technology in reconstructive surgery. J Surg Oncol $118(5): 845-852$

29. Tarsitano A, Battaglia S, Crimi S, Ciocca L, Scotti R, Marchetti C (2016) Is a computer-assisted design and computer-assisted manufacturing method for mandibular reconstruction economically viable? J Craniomaxillofac Surg 44(7):795-799

30. Zweifel DF, Simon C, Hoarau R, Pasche P, Broome M (2015) Are virtual planning and guided surgery for head and neck reconstruction economically viable? J Oral Maxillofac Surg 73(1):170-175

31. Mazzola F, Smithers F, Cheng K, Mukherjee P, Hubert Low TH, Ch'ng S et al (2020) Time and cost-analysis of virtual surgical planning for head and neck reconstruction: a matched pair analysis. Oral Oncol 01(100):104491

32. Morone PJ, Shah KJ, Hendricks BK, Cohen-Gadol AA (2019) Virtual, 3-dimensional temporal bone model and its educational value for neurosurgical trainees. World Neurosurg 01(122):e1412-e1415

33. Fang TY, Wang PC, Liu CH, Su MC, Yeh SC (2014) Evaluation of a haptics-based virtual reality temporal bone simulator for anatomy and surgery training. Comput Methods Programs Biomed 113(2):674-681

34. Stepan K, Zeiger J, Hanchuk S, Del Signore A, Shrivastava R, Govindaraj S et al (2017) Immersive virtual reality as a teaching tool for neuroanatomy. Int Forum Allergy Rhinol 7(10): 1006-1013

35. Ziemkiewicz C, Ottley A, Crouser RJ, Chauncey K, Su SL, Chang $R$ (2012) Understanding visualization by understanding individual users. IEEE Comput Graphics Appl 32(6):88-94

36. Nguyen N, Mulla A, Nelson AJ, Wilson TD (2014) Visuospatial anatomy comprehension: the role of spatial visualization ability and problem-solving strategies. Anat Sci Educ 7(4):280-288

37. Weech S, Kenny S, Barnett-Cowan M (2019) Presence and cybersickness in virtual reality are negatively related: a review. Front Psychol 04(10):158

38. Vincenzi DA, Wise JA, Mouloua M, Hancock PA. Human factors in simulation and training. CRC Press; 2008.

39. Stanney KM, Hale KS, Nahmens I, Kennedy RS (2003) What to expect from immersive virtual environment exposure: influences of gender, body mass index, and past experience. Hum Factors 45(3):504-520

40. Creighton FX, Unberath M, Song T, Zhao Z, Armand M, Carey J (2020) Early feasibility studies of augmented reality navigation for lateral skull base surgery. Otol Neurotol 41(7):883-888

41. Wong K, Yee HM, Xavier BA, Grillone GA (2018) Applications of augmented reality in otolaryngology: a systematic review. Otolaryngol Head Neck Surg. https://doi.org/10.1177/0194599818 796476

Publisher's Note Springer Nature remains neutral with regard to jurisdictional claims in published maps and institutional affiliations. 